\title{
FORCED CONVECTION IN DUCTS WITH PERMEABLE WALLS
}

\author{
C. H. Alexandrino \\ and M. L Martins-Costa \\ Universidade Federal Fluminense \\ Departamento de Engenharia Mecânica \\ Laboratório de Mecânica Teórica e Aplicada \\ Rua Passo da Pátria, 156 \\ 24210-240 Niterói/RJ, Brazil \\ ABSTRACT \\ A mixture theory model is employed in a local description of the energy transfer \\ in a duct with permeable wall which is simulated by considering two distinct flow \\ regions, one consisting of a Newtonian incompressible fluid and the other \\ represented by a binary (solid-fluid) mixture. Compatibility conditions at the \\ interface (pure fluid-mixture) for momentum and energy transfer are considered. \\ The simulations are carried out by using a finite difference approach with an \\ upwind strategy for the convective term discretization. \\ laura@mec.uff.br
}

\section{INTRODUCTION}

Ducts with permeable walls are present in many relevant engineering situations such as flow of perforation mud in oil wells and porous bearing lubrication. This work employs a Mixture Theory approach to model the momentum and energy transfer in a permeable duct by considering two distinct flow regions. The former inside the duct where a Newtonian incompressible fluid flows and the latter representing the duct's permeable wall - a fluidsaturated porous medium, from now on referred to as mixture region. In this region both fluid and porous matrix are treated as overlapping continuous constituents of a binary mixture. The fluid constituent and the fluid are assumed Newtonian and incompressible while the solid constituent, representing the porous matrix, is assumed rigid, homogeneous, isotropic and at rest.

Most of the works dealing with transport in porous media employ a local volume-averaging technique to describe quantities such as temperature, pressure, concentration and the velocity components; allowing the use of the classical Continuum Mechanics approach. Vafai and Kim (1990) have used this approach and Darcy's law combined with Brinkmann and Forchheimer terms to account for inertia and viscous effects and satisfy the noslip condition - as the balance of linear momentum to analyze convection in two distinct flow regions (fluid and fluid-saturated porous medium). Vafai and Sozen (1990) have considered thermal nonequilibrium (a natural assumption in a Mixture Theory approach) with a local volume-averaging technique, considering Erguns' correlation as the vapor phase momentum equation, in order to analyze the forced convective flow of a gas through a packed-bed of solid particles. Alazmi and Vafai (2000) have analyzed volume averaging models for transport through porous media by considering four categories of processes: constant porosity models, variable porosity models, including thermal dispersion (combined with variable porosity or not) and models allowing for local thermal nonequilibrium.

A distinct approach is used in this work: the Continuum Theory of Mixtures (Bedford and Drumheller, 1983, Rajagopal and Tao, 1995) - supported by a local theory generalizing the classical Continuum
Mechanics (Gurtin, 1981), specially developed to describe multiphase phenomena. It models fluid saturated porous media by considering the fluid and the porous matrix as superimposed continuous constituents of a binary mixture - each of them occupying its whole volume. The mixture theory allows the existence of $n$ distinct velocity fields and $n$ distinct temperature fields requiring additional terms, playing the role of momentum and energy sources, to account for the thermomechanical coupling among the constituents in the balance equations. Thermodynamically consistent constitutive relations for these sources are used (Martins-Costa et al., 1992; Costa Mattos et al., 1995).

Compatibility conditions at the interface (pure fluid-mixture) for momentum and energy transfer must be imposed in order to allow the solution of the problem. These conditions have been considered supposing no flow across the interface (Martins-Costa et al., 1994). Starting from known velocity fields, the forced convection heating of a fluid flowing through the permeable duct is simulated by using a finite difference approach. A central finite difference discretization is used for the diffusive terms while an upwind strategy (Euvrard, 1987) is employed for the convective terms discretization.

(1) Graduate student at Mechanical Engineering Program (PGMEC)/UFF $\left.{ }^{2}\right)$ All article correspondence should be addressed to this author.

\section{MECHANICAL MODEL}

Representing the regions occupied by the pure fluid and the mixture, respectively, by the open sets $\Omega_{1}$ and $\Omega_{2}$, with boundaries $\partial \Omega_{1}$ and $\partial \Omega_{2}$, the mass balance, in the absence of mass production by any constituent in $\Omega_{2}$, may be written as (Gurtin, 1981; Rajagopal and Tao, 1995)

$$
\begin{array}{ll}
\frac{\partial \rho}{\partial t}+\nabla \cdot(\rho \mathbf{v})=0 & \text { in } \Omega_{1} \\
\frac{\partial \rho_{i}}{\partial t}+\nabla \cdot\left(\rho_{i} \mathbf{v}_{i}\right)=0 & \text { in } \Omega_{2}
\end{array}
$$




\section{CIÊNCIA/SCIENCE}

in which the actual fluid parameters - defined in $\Omega_{1}-$ are $\rho$ standing for its mass density and $\mathbf{v}$ for its velocity. In the mixture region $\Omega_{2}$, balance equations must hold for $i=1, n$ constituents. The $i$-th constituent parameters are $\rho_{i}$, its mass density - representing locally the ratio between its mass and the respective volume of mixture and $\mathbf{v}_{i}$ standing for its velocity. The momentum balance is given by (Gurtin, 1981; Rajagopal and Tao, 1995)

$$
\begin{array}{ll}
\rho\left[\frac{\partial \mathbf{v}}{\partial t}+(\nabla \mathbf{v}) \mathbf{v}\right]=\nabla \cdot \mathbf{T}+\rho \mathbf{g}, & \text { in } \Omega_{1} \\
\rho_{i}\left[\frac{\partial \mathbf{v}_{i}}{\partial t}+\left(\nabla \mathbf{v}_{i}\right) \mathbf{v}_{i}\right]=\nabla \cdot \mathbf{T}_{i}+\mathbf{m}_{i}+\rho_{i} \mathbf{g}, & \text { in } \Omega_{2}
\end{array}
$$

where $\mathbf{T}$ represents Cauchy stress tensor (in $\Omega_{1}$ ) and $\mathbf{T}_{i}$ is the partial stress tensor associated with the $i$-th constituent (in the mixture region $\Omega_{2}$ ). The body force is represented by $\mathbf{g}$ everywhere while $\mathbf{m}_{i}$, defined in the mixture region $\Omega_{2}$, is the momentum supply due to the interaction between the $\mathrm{i}$-th constituent and the remaining constituents. It arises from the possible existence of $n$ distinct velocity fields in an $n$-constituents mixture at each spatial point, so that the net momentum supply to the mixture is zero, in other words, $\sum_{i=1}^{n} \mathbf{m}_{i}=0$ (in $\Omega_{2}$ ).

The conservation of energy in both regions $\Omega_{1}$ and $\Omega_{2}$ is expressed as (Gurtin, 1981; Rajagopal and Tao, 1995; Martins-Costa et al., 1993)

$$
\begin{aligned}
\rho c\left[\frac{\partial T}{\partial t}+(\nabla T) \mathbf{v}\right]= & -\nabla \cdot \mathbf{q}+\mathbf{T} \cdot \mathbf{D}+q^{\prime \prime \prime}, \text { in } \Omega_{1} \\
\rho_{i} c_{i}\left[\frac{\partial T_{i}}{\partial t}+\left(\nabla T_{i}\right) \mathbf{v}_{i}\right] & =-\nabla \cdot \mathbf{q}_{i}+\mathbf{T}_{i} \cdot \mathbf{D}_{i}+ \\
& +q_{i}^{\prime \prime \prime}+\psi_{i}, \quad \text { in } \Omega_{2}
\end{aligned}
$$

where the parameters defined in the region $\Omega_{1}$ are $T$, representing the fluid temperature, $c$, its specific heat, $\mathbf{D}$, the symmetrical part of $\nabla \mathbf{v}$ (the fluid velocity gradient) in such a way that the viscous dissipation is given by the inner product $\mathbf{T} \cdot \mathbf{D}$. The fluid heat flux vector is given by $\mathbf{q}$ and, finally, the external heat supply to the fluid is $q^{\prime \prime \prime}$. In the mixture region $\Omega_{2}$, the $i$-th constituent temperature is given by $T_{i}$, the partial heat flux associated with the $i$-th constituent by $\mathbf{q}_{i}$, the symmetrical part of $\nabla \mathbf{v}_{i}$ by $\mathbf{D}_{i}$ and the external heat supply to the $i$-th constituent is $q_{i}^{\prime \prime \prime}$. Besides, $c_{i}$ represents the $i$-th constituent specific heat, measured in a continuum mechanics context and $\psi_{i}$ an internal heat source, defined in $\Omega_{2}$, which arises from the possible existence of $n$ distinct temperature fields in an $n$ constituents mixture at each spatial point. It represents the $i$-th constituent thermal interaction with the remaining constituents of the mixture in such a way that

\section{H. Alexandrino et al. Forced Convection...}

the following equation must hold (Martins-Costa et al., 1992; 1993): $\sum_{i=1}^{n} \psi_{i}=0\left(\right.$ in $\left.\Omega_{2}\right)$.

Constitutive relations for the stress tensor in $\Omega_{1}$ and the partial stress tensor and momentum source in $\Omega_{2}$ are required to solve the hydrodynamic problem considering a Newtonian fluid flowing in a domain $\Omega_{1} \cup \Omega_{2}$ (see Martins-Costa et al., 1994 and references therein).

$$
\begin{array}{ll}
\mathbf{T}=-p \mathbf{I}+2 \mu \mathbf{D} & \text { in } \Omega_{1} \\
\mathbf{T}_{F}=-p \varphi \mathbf{I}+2 \lambda \varphi^{2} \mu \mathbf{D}_{F} & \text { in } \Omega_{2} \\
\mathbf{m}_{F}=-\frac{\varphi^{2} \mu}{K} & \text { in } \Omega_{2}
\end{array}
$$

In equations (4) $p$ is the pressure, $\mu$ the actual fluid viscosity, $\varphi$ and $K$ are the porous matrix porosity and specific permeability, $\lambda$ is a scalar parameter depending on the porous matrix microstructure and the index $F$ refers to the fluid constituent.

In order to build a mechanical model for the duct with permeable wall, constitutive relations are yet required for the heat flux $\mathbf{q}$ in $\Omega_{1}$ and for the solid and fluid constituents' partial heat fluxes $\mathbf{q}_{S}$ and $\mathbf{q}_{F}$ and internal supplies $\psi_{S}$ and $\psi_{F}$ in $\Omega_{2}$ (Martins-Costa et al., 1994)

$$
\begin{array}{ll}
\mathbf{q}=-k_{f} \nabla T & \text { in } \Omega_{1} \\
\mathbf{q}_{F}=-\Lambda k_{f} \varphi \nabla T_{F} & \text { in } \Omega_{2} \\
\mathbf{q}_{S}=-\Lambda k_{s}(1-\varphi) \nabla T_{S} & \text { in } \Omega_{2} \\
\psi_{F}=-\psi_{S}=R_{F S}\left(T_{S}-T_{F}\right) & \text { in } \Omega_{2}
\end{array}
$$

in which $k_{f}$ is the Newtonian fluid thermal conductivity and $k_{s}$ is the porous matrix thermal conductivity, $\Lambda$ represents a scalar positive-valued parameter which may depend on both the internal structure and the kinematics of the mixture and $R_{F S}$ is a positive-valued factor which depends not only on spatial position and on both constituents' thermal properties but also on their velocities, accounting for the convective heat transfer.

At the interface between the regions $\Omega_{1}$ and $\Omega_{2}-$ defined by the set $\partial \Omega_{I} \equiv \bar{\Omega}_{1} \cap \bar{\Omega}_{2}-$ compatibility conditions must be imposed (Martins-Costa et al, 1994)

$$
\begin{aligned}
& \mathbf{v}=\varphi \mathbf{v}_{F} \\
& \varphi \mathbf{T n} \cdot \mathbf{t}=\mathbf{T}_{F} \mathbf{n} \cdot \mathbf{t}
\end{aligned}
$$

in which $\mathbf{n}$ is a unit outward normal to $\partial \Omega_{I}$ and $\mathbf{t}$ is any tangent to $\partial \Omega_{I}$. The compatibility equations (6) have been obtained from the solution of thermodynamically consistent equations in both regions (see Martins-Costa et al., 1994 and references therein). They do not suffer from the difficulty of matching the porous medium flow equations with the Navier-Stokes equation, discussed by Nield and Bejan (1992).

In order to assure continuity of the temperature field and continuity and adequate distribution of the normal 


\section{CIÊNCIA/SCIENCE}

heat flux throughout the interface, the following compatibility conditions are imposed at $\partial \Omega_{I}$ (MartinsCosta et al, 1994):

$$
\begin{array}{lr}
T=\varphi T_{F}+(1-\varphi) T_{S} & \text { at } \partial \Omega_{I} \\
\varphi \mathbf{q} \cdot \mathbf{n}=\mathbf{q}_{F} \cdot \mathbf{n} & \text { at } \partial \Omega_{I} \\
(1-\varphi) \mathbf{q} \cdot \mathbf{n}=\mathbf{q}_{S} \cdot \mathbf{n} & \text { at } \partial \Omega_{I}
\end{array}
$$

Vafai and Kim (1990) have used similar conditions, imposing continuity of temperature and heat flux fields at the interface.

\section{CONVECTION IN PERMEABLE DUCTS}

Considering the porous matrix rigid and at rest it suffices to solve mass and momentum balances for the fluid constituent in the mixture region, while both constituents in this region must satisfy the energy balance. The regions composing the permeable duct considered in this work are defined as $\Omega_{1} \equiv\{(r, z)$ such that $\left.0<r<R_{1}, \quad 0<z<L\right\}$ and $\Omega_{2} \equiv\{(r, z)$ such that $\left.R_{1}<r<R_{2} \quad, 0<z<L\right\} \quad$ and the interface as $\partial \Omega_{I} \equiv\left\{(r, z)\right.$ such that $\left.r=R_{1}, 0<z<L\right\}$.

Supposing the flow not affected by the thermal problem, the velocity fields in $\Omega_{1}$ and $\Omega_{2}$ may be analytically obtained, provided that a steady-state fully developed axisymmetric flow is considered. Denoting by $v$ and $v_{F}$ the $z$-components of $\mathbf{v}$ and $\mathbf{v}_{F}$ and taking into account that $\rho_{F}=\varphi \rho_{f}$ the hydrodynamic problem is represented by

$$
\begin{aligned}
& \frac{\partial \phi}{\partial z}+\mu \frac{1}{r} \frac{\partial}{\partial r}\left(r \frac{\partial v}{\partial r}\right)=0 \\
& 0 \leq r<R_{1} \\
& \varphi \frac{\partial \phi}{\partial z}+\mu \varphi^{2} \lambda \frac{1}{r} \frac{\partial}{\partial r}\left(r \frac{\partial v_{F}}{\partial r}\right)-\frac{\varphi^{2} \mu}{K} v_{F}=0, R_{1}<r<R_{2} \\
& \phi \equiv-p+\rho g_{z} z \quad \Rightarrow \quad \frac{\partial \phi}{\partial z}=-\frac{\partial p}{\partial z}+\rho g_{z} \\
& v \text { finite } \forall r \\
& v_{F}=0 \quad r=R_{2} \\
& \varphi v_{F}=v \quad r=R_{1} \\
& \lambda \varphi \frac{\partial v_{F}}{\partial r}=\frac{\partial v}{\partial r} \quad r=R_{1}
\end{aligned}
$$

with $0<z<L$. In equations (8) the boundary conditions are represented by the fourth and fifth equations - the former being employed to assure that the velocity must remain finite at $r=0$ and the latter representing the classical no-slip condition at the impermeable interface located at $r=R_{2}$. The compatibility of velocity and momentum transport across the interface located at $r=R_{1}$ is represented in the last two equations. The solution of equations (8) is (Saldanha da Gama and Sampaio, 1983)

$$
\begin{aligned}
v= & \frac{K}{\mu} \frac{\partial \phi}{\partial z}\left\{\frac{\lambda}{4}\left(\chi_{1}^{2}-\chi^{2}\right)+1-\psi_{I 0}-\frac{\psi_{K I}}{\psi_{I K}}\left(\psi_{I}-\frac{\chi_{1}}{2}\right)\right\} \\
v_{F}= & \frac{K}{\mu \varphi} \frac{\partial \phi}{\partial z}\left\{1-\frac{\mathrm{I}_{0}(\chi)}{\mathrm{I}_{0}\left(\chi_{2}\right)}+\left(\frac{\chi_{1}}{2}-\psi_{I}\right) \times\right. \\
& \left.\times\left(\frac{\mathrm{K}_{0}(\chi) \mathrm{I}_{0}\left(\chi_{2}\right)-\mathrm{K}_{0}\left(\chi_{2}\right) \mathrm{I}_{0}(\chi)}{\psi_{I K}}\right)\right\} \\
\chi= & \frac{r}{\sqrt{K \lambda}} \quad \chi_{1}=\frac{R_{1}}{\sqrt{K \lambda}} \quad \chi_{2}=\frac{R_{2}}{\sqrt{K \lambda}} \\
\psi_{I 0}= & \frac{\mathrm{I}_{0}\left(\chi_{1}\right)}{\mathrm{I}_{0}\left(\chi_{2}\right)} \quad \psi_{I}=\frac{\mathrm{I}_{1}\left(\chi_{1}\right)}{\mathrm{I}_{0}\left(\chi_{2}\right)} \\
\psi_{K I}= & \mathrm{K}_{0}\left(\chi_{1}\right) \mathrm{I}_{0}\left(\chi_{2}\right)-\mathrm{K}_{0}\left(\chi_{2}\right) \mathrm{I}_{0}\left(\chi_{1}\right) \\
\psi_{I K}= & \mathrm{I}_{0}\left(\chi_{2}\right) \mathrm{K}_{1}\left(\chi_{1}\right)+\mathrm{I}_{1}\left(\chi_{1}\right) \mathrm{K}_{0}\left(\chi_{2}\right)
\end{aligned}
$$

with the fluid velocity $v$ defined for $0<r<R_{1}$ and the fluid constituent velocity $v_{F}$ defined for $R_{1}<r<R_{2}$ and $\mathrm{I}_{0}, \mathrm{I}_{1}, \mathrm{~K}_{0}$ and $\mathrm{K}_{1}$ representing modified Bessel functions. It is important to notice that for the limiting case in which the permeability $K \rightarrow 0$ a classical HagenPoiseuille flow is recovered for the fluid velocity $v$, since $\chi^{2} K \lambda=r^{2}$.

Assuming the external sources $q^{\prime \prime \prime}$ and $q_{i}^{\prime \prime \prime}$ equal to zero and neglecting $\mathbf{T} \cdot \mathbf{D}$ and $\mathbf{T}_{F} \cdot \mathbf{D}_{F}$, the energy equations reduce to the system described by equations (10), subjected to the boundary conditions (11) and compatibility conditions (12), provided that the following variables are defined

$$
\begin{aligned}
\alpha=\frac{\rho_{f} c_{f}}{k_{f}} & \alpha_{F}=\frac{\rho_{f} c_{f}}{\Lambda k_{f}} \\
\beta_{F}=\frac{R_{F S}}{\Lambda k_{f} \varphi} & \beta_{S}=\frac{R_{F S}}{\Lambda k_{s}(1-\varphi)}
\end{aligned}
$$

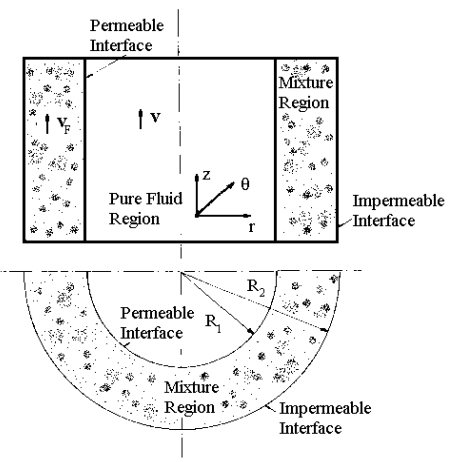

Figure 1. Problem scheme. 


$$
\begin{aligned}
& T=T_{0} \quad 0<r<R_{1} \quad z=0 \\
& T_{F}=T_{F 0} \quad R_{1}<r<R_{2} \quad z=0 \\
& \frac{\partial T_{S}}{\partial z}=0 \quad R_{1}<r<R_{2} \quad z=0 \\
& T \text { finite } \forall r \quad 0<z<L \\
& \frac{\partial T_{F}}{\partial r}=0 \quad r=R_{2} \quad 0<z<L \\
& \frac{\partial T_{S}}{\partial r}=0 \quad r=R_{2} \quad 0<z<L \\
& \frac{\partial T}{\partial z}=0 \quad 0<r<R_{1} \quad z=L \\
& \frac{\partial T_{F}}{\partial z}=0 \quad R_{1}<r<R_{2} \quad z=L \\
& \frac{\partial T_{S}}{\partial z}=0 \quad R_{1}<r<R_{2} \quad z=L \\
& T=\varphi T_{F}+(1-\varphi) T_{S}, r=R_{1}, 0<z<L \\
& \frac{\partial T}{\partial r}=\Lambda \frac{\partial T_{F}}{\partial r}, \quad r=R_{1}, 0<z<L \\
& \frac{\partial T}{\partial r}=\Lambda \frac{k_{s}}{k_{f}} \frac{\partial T_{S}}{\partial r}, \quad r=R_{1}, 0<z<L
\end{aligned}
$$

\section{NUMERICAL RESULTS}

Since no analytical solution for the heat transfer problem - given by equations (10)-(12) is known, a finite difference approach (Euvrard, 1987) is employed to find numerical approximations to its solution. For the diffusive terms, a central finite difference scheme discretization was used, while an upwind scheme was employed in the convective term discretization. As the temperature coefficients matrix associated to the system of equations (10) is sparse, a grid description, in which each temperature (fluid and fluid and solid constituents) possesses two indexes, according to its position on the grid, is used. Each iteration, represented in such a way to allow an effective storage scheme with memory reutilization, is then solved with the help of the GaussSeidel method.

In this section some results, considering a long permeable duct with a fixed geometry - namely a duct with unit external radius and 100 units length, are presented.

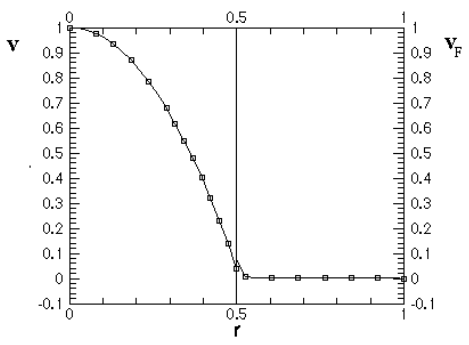

Figure 2. Fluid and fluid constituent velocity profiles.

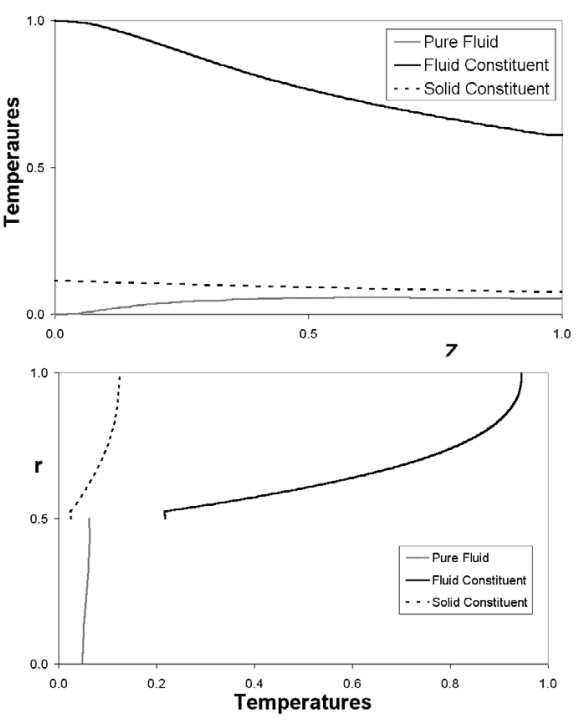

Figure 3. Fluid temperature $\left(r=r_{a}\right)$ and fluid and solid constituents temperatures $\left(r=r_{b}\right)$ along $z$. Temperature profiles (central section). $\alpha=\alpha_{F}=10^{5}, \beta_{F}=\beta_{S}=10^{0}$.

Figure 1 shows the geometry of the problem characterized by velocity profiles given by equation (9) and temperatures satisfying equations (10) to (12). In Figure 2 velocity profiles for the pure fluid and the fluid constituent - the former flowing in the region inside the duct $0<r<R_{1}$ and the latter flowing in the porous region - given by $R_{1}<r<R_{2}$ are presented, considering Darcy number - defined as the ratio between the porous matrix permeability and the square of a characteristic dimension $D a=K / R_{2}^{2}$ given by $D a=10^{-4}$. The velocities are such that $v=v / \frac{K}{\mu} \frac{\partial \phi}{\partial z}$ and $v_{F}=v_{F} / \frac{K}{\mu \varphi} \frac{\partial \phi}{\partial z}$.
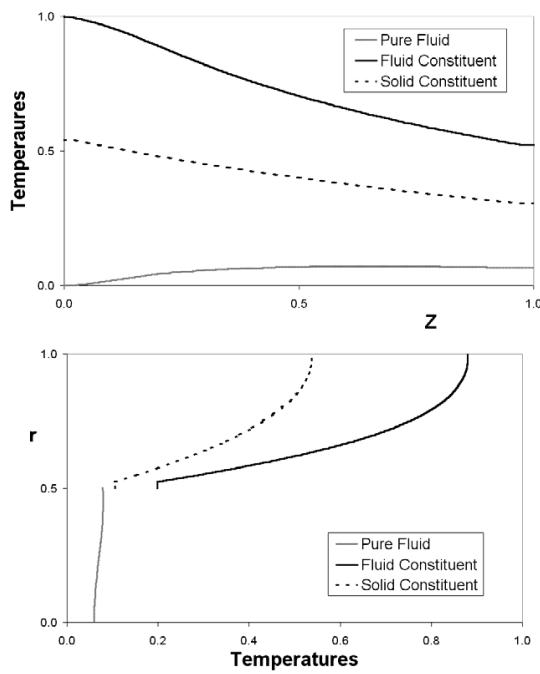

Figure 4. Fluid temperature $\left(r=r_{a}\right)$ and fluid and solid constituents temperatures $\left(r=r_{b}\right)$ along $z$. Temperature profiles (central section). $\alpha=\alpha_{F}=10^{5}, \beta_{F}=\beta_{S}=10^{1}$. 


\section{CIÊNCIA/SCIENCE}

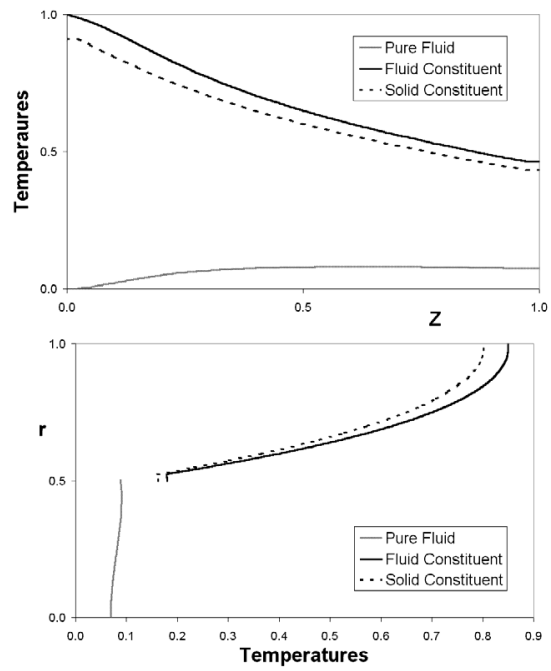

Figure 5. Fluid temperature $\left(r=r_{a}\right)$ and fluid and solid constituents temperatures $\left(r=r_{b}\right)$ along $z$. Temperature profiles (central section). $\alpha=\alpha_{F}=10^{5}, \beta_{F}=\beta_{S}=10^{2}$

Figures 3 to 6 present - at the top - the temperature behavior for the fluid flowing inside the porous duct (in the so-called pure fluid region) by considering a central section characterized by $r_{a}=R_{1} / 2$ as well as the thermal behavior of both the fluid and solid constituents, depicted in a central section of the porous region - namely at $r_{b}=R_{1}+\left(R_{2}-R_{1}\right) / 2$, both versus longitudinal coordinate $z$. At the bottom, they show temperature profiles at a cross section located in the middle of the porous duct $(z=L / 2)$ in which the pure fluid occupies the region $0<r<R_{1}$ and the fluid and the solid constituents coexist superposed in the region $R_{1}<r<R_{2}$. All these figures have been obtained by considering the fluid inlet temperature $T_{0}=0$ in the fluid region $\Omega_{1}$ and the fluid constituent inlet temperature $T_{F 0}=1$ in the mixture region $\Omega_{2}$ - representing the permeable duct. Besides, in all the shown examples a unitary ratio of heat conduction between both constituents - namely $k_{f} \varphi / k_{s}(1-\varphi)=1$ has been considered. It is worth of notice that two distinct temperature profiles are shown in the permeable duct region, since thermal nonequilibrium has been assumed.
C. H. Alexandrino et al. Forced Convection...
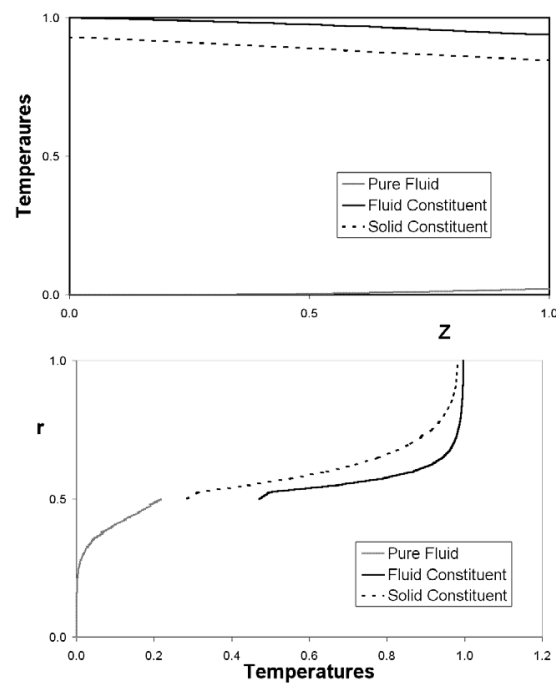

Figure 6. Fluid temperature $\left(r=r_{a}\right)$ and fluid and solid constituents temperatures $\left(r=r_{b}\right)$ along $z$. Temperature profiles (central section). $\alpha=\alpha_{F}=10^{6}, \beta_{F}=\beta_{S}=10^{2}$.

Figures 3 to 5 show the influence of the $\beta_{i}$ parameter on the temperature behavior. This parameter represents a ratio of the heat transfer between both solid and fluid constituents - taken into account in the energy generation term $\psi_{i}$ that plays the role of an internal heat source to the $i$-th constituent heat conduction. A decrease in the $\beta_{i}$-parameter results in a tendency to a common value for both the solid and the fluid constituents' temperatures in the porous region. In other words, as $\beta_{i}$ decreases the tendency toward thermal equilibrium between both constituents increases.

The influence of the thermal diffusivity represented by a parameter $1 / \alpha$ in the pure fluid region and redefined by a parameter $1 / \alpha_{F}$ in the mixture region where $\Lambda$ has been included to account for the mixture structure and kinematics - may be noticed by comparing Figures 5 and 6.

As the $\alpha$-parameter increases (and the thermal diffusivity decreases) both constituents' temperatures at a central section $r=r_{b}$ in the porous region tend to the fluid constituent inlet temperature $\left(T_{F 0}=1\right)$ while in the pure fluid region the fluid temperature at $r=r_{a}$ tend to the fluid inlet temperature $\left(T_{0}=0\right)$. An effect of the inlet boundary condition being carried out through the flow is suggested by this behavior. This effect is more pronounced as the $\alpha$-parameter increases.

\section{FINAL REMARKS}

A mixture theory model has been employed to describe the momentum and energy transport in a permeable duct with thermal nonequilibrium being allowed. Two distinct flow regions were considered - in the porous region (the duct), fluid and solid (the porous matrix) were treated as continuous constituents of a binary mixture while the classical continuum mechanics equations have been recovered in the pure fluid region - 
namely the region inside the duct, where the mixture was reduced to a single constituent. Adequate conditions at the interface pure fluid-mixture were imposed.

\section{ACKNOWLEDGEMENTS}

The author M.L. Martins-Costa gratefully acknowledges the financial support provided by Brazilian agency CNPq through grant 300404/91-3.

\section{REFERENCES}

Alazmi, B. and Vafai, K., 2000, Analysis of Variants Within the Porous Media Transport Models, J. Heat Transfer, Vol. 122, pp. 303-326.

Bedford, A. and Drumheller, D.S., 1983, Recent advances - Theories of immiscible and structured mixtures, Int. J. Engng. Sci., Vol. 21, No. 8, pp. 863-960.

Costa-Mattos, H., Martins-Costa, M.L. and Saldanha da Gama, R.M., 1995, On the Modelling of Momentum and Energy Transfer in Incompressible Mixtures, Int. J. Non-Linear Mechanics, Vol. 30, No. 4, pp. 419-431.

Euvrard, D., 1987, Résolution Numérique des Équations aux Dérivées Partielles, Masson, Paris.

Gurtin, M.E., 1981, An introduction to continuum mechanics, Academic Press, New York.

Martins-Costa, M.L., Saldanha da Gama, R.M. and Sampaio, R., 1992, Modelling and Simulation of Energy Transfer in a Saturated Flow through a Porous Medium, Appl. Math. Modelling, Vol. 13, No. 4, pp. 589-597.

Martins-Costa, M.L., Saldanha da Gama, R.M. and Sampaio, R., 1993, On the Energy Balance for Continuous Mixtures, Mech. Research Comm., Vol. 20, No. 1, pp. 53-58.

Martins-Costa, M.L., Saldanha da Gama, R.M. and Sampaio, R., 1994, A Local Model for the Heat Transfer Process in Two Distinct Flow Regions, Int. J. Heat Fluid Flow, Vol. 15, No. 6, pp. 477-485.

Nield, D.A. and Bejan, A., 1992, Convection in Porous Media, Springer-Verlag, New York.

Rajagopal and Tao, 1995, Mechanics of Mixtures, Series on Advances in Mathematics for Applied Sciences, Vol. 35, World Scientific, Singapore.

Saldanha da Gama, R.M. and Sampaio, R., 1983, Modelling for Two-Regions Flows, One Occupied by a Viscous Fluid and the other by a Mixture, J. Braz. Soc. Mech. Sci., Vol. 5, No. 1, pp. 3-17.

Vafai, K. and Kim, S.J., 1990, Analysis of surface enhancement by a porous substrate, J. Heat Transfer, Vol. 112, pp. 700-706.

Vafai, K. and Sozen, M., 1990, Analysis of Energy and Momentum Transport for Fluid Flow through a Porous Bed, J. Heat Transfer, Vol. 112, pp. 690-699. 